\title{
DISTRIBUTION PATTERN OF UNDERWATER ILLUMINANCE FOR SQUID NET FISHERIES IN MALAYSIA
}

\author{
Sakri Ibrahim*, Mohd. Azmi Ambak*', Khalid Samo*, \\ Mohamad Zaidi Zakaria* ${ }^{*}$, Zainal Ashirin Shahardin ${ }^{*}$ and Sukree Hajisamae
}

\begin{abstract}
A study on the distribution pattern of underwater illuminance for squid net fisheries in Malaysia was conducted from April to September, 1996 in sheltered waters of Kapas Island off the coast of Terengganu. Peninsular Malaysia at Latitude $5^{\circ} 13.6^{\prime} \mathrm{N}$ and Longitude $103^{\circ} 15.8^{\prime} \mathrm{E}$. Three sets of parameters were collected from three commercial squid fishing boats and underwater illuminance as estimated using a theoretical model. It was found that the underwater illuminance from the lighting systems of all the three boats managed to reach a depth of more than $40 \mathrm{~m}$ (maximum depth of the fishing ground is $22 \mathrm{~m}$ ). High Pressure Mercury lamps were found to produce higher lighting efficiencies as compared to incandescent lamps. Squid net fishermen in the study urea were found to be employing excess power for the fishing operation.
\end{abstract}

KEYWORDS: underwater illuminance, squid fishing, lighting systems.

\section{INTRODUCTION}

Artificial light has long been used in exploitation of commercial fish species (including cephalopods) in all parts of the world. This method has been developed empirically and the intensity of light has been increased without any due consideration to the theoretical knowledge of fish attraction by light (Kawamura et al., 1983; Hayase et al., 1983). The increase in the power of light intensity over the optimum limitation has become a serious concern for marine biologists (Nomura, 1985). Squid fishermen argue that the increase of the power of fishing lights is necessary to attract more squid in their effort to compete with other fishermen operating in the same fishing ground

In Malaysia, squid is mainly landed by trawls, squid nets, squid jigs, purse seines and traps (Sakri et al., 1995). With the exception of trawls, other gears are usually operated at nighttime with the use of artificial light onboard the fishing boat as a means to aggregate squid for successful harvesting operations (Ashirin \& Ibrahim, 1992). Squid nets are one of the most popular squid fishing gears in Malaysia especially in the states of Kelantan and Terengganu (on the east coast of Peninsular Malaysia). The gear is operated only at nighttime especially during moonless nights (after or before new moon) by taking advantage of the squid response to artificial light. This gear is very efficient at catching squids and has great potential in the near future. There are two types of lighting systems installed onboard squid netting boats that are used with the squid net; the attracting and the controllable light systems. However, this study concentrated only on the attracting light system which is vital in aggregating squid before the harvesting operations. Knowledge of underwater light distribution patterns is important for the success and development of squid capture fisheries. Apart from the reaction pattern of squid to light, the physical factors such as water transparency that affect underwater illuminance should also be considered in order to improve the catching efficiency and reduce the energy consumption of the squid fishing boats. Underwater illuminance is often very difficult to obtain by direct measurement due to the problems related to sea conditions that researchers encounter during their study. However, under-water illuminance can be estimated theoretically using a model as has been described by Hajisamae (1996), Hamid (1990) and Ogura et al. (1985). The purpose of this study is thus to determine the distribution pattern of underwater illuminance of Malaysian squid netting boats using the theoretical model based on Ogura's method.

\section{MATERIALS AND METHODS}

Ogura et al. (1985) described a theoretical model for the estimation of underwater illuminance. According to the model, underwater illuminance

\footnotetext{
* Faculty of Applied Science \& Technol., Universiti Putra Malaysia Terengganu, Malaysia

**, Faculty of Science \& Technol., Prince of Songkla University, Thailand
} 
(I ) at a layer $r$ meters below the sea surface may be calculated using the following formula

$$
I \pi=I \phi \frac{A \Phi}{A \pi} k \pi e^{-\mu r}
$$

where;

$\bar{\omega}=$ incident angle

$\phi \quad=$ refraction angle

$\mathrm{I} \phi=\mathrm{C}=$ total lumen of light source $/ 4 \mu$

$\mathrm{Ak}=$ areas of wave front at $1 \mathrm{~m}$ below the light source

$A \omega=$ areas of wave front at $r \mathrm{~m}$ below the sea water

$\mathrm{k} \omega=$ =light transmission with a nearly constant value of 0.98

$\mu \quad=$ attenuation coefficient of light in sea water $=1.7 /$ transparency

$r \quad=$ a layer $r$ meter below the surface of the sea $(\mathrm{m})$

$\mathrm{n} \quad=\sin / \sin$

=an almost constant value of 1.33

h =average height of light source above sea level (m)

The formula for practical application is given below:

$$
I \pi=\frac{k \pi \cdot e^{-\mu r} \cdot c}{e^{2}\left[\frac{1}{\cos \phi}+\frac{r}{n h}\right] \times\left[\frac{\cos \pi}{\cos \phi}+\frac{r \cos \phi}{n h \cos \pi}\right]}
$$

Parameters required for the estimation of underwater illuminance according to the theoretical model as described by Ogura et al. (1985) were taken from three commercial squid fishing boats operating in Peninsular Malaysian coastal waters of the South China Sea. For an estimation of the underwater illuminance, three sets of parameters were recorded from the three boats.

\section{a. Total candela of light source}

The total candela (C) of light source was estimated based on the types, the numbers and the power of lamps on each boat. Total lumen of light was initially estimated by multiplying the total power of light source (watt) by lighting efficiency; 37.5 lumen/watt for high pressure mercury lamp and 19.2 lumen/watt for incandescent lamp (Nomura, 1985). The obtained value was then converted to total candela using the following formula:

Total candela $=$ total lumen $/ 4$

\section{b. Average height of light source above the sea level}

The height of the light source was taken as the vertical distance from the light source to the sea level. This parameter was measured from the three selected boats.

\section{c. Water transparency}

Water transparency was used to calculate the attenuation coefficient of light in water (new) of the selected fishing ground which was then approximated using the formula of $\mu=1.7 /$ transparency (Ogura et al., 1985). Based on the average transparency of about $13 \mathrm{~m}$ (the regional transparency in this fishing ground is $10-15 \mathrm{~m}$ ), the light attenuation in water for this fishing ground is computed to be approximately 0.13

In addition to the above parameters, refraction of light in water also determines the intensity of underwater illuminance. The relationship between the angle of incidence to the angle of refraction for sea water is given in Table 1 .

Table 1. Relationship between angle of incident $(\phi)$ and angle of refraction (i) (after Nomura, 1985).

\begin{tabular}{cc}
\hline $\begin{array}{c}\text { Incident angle } \\
(\varnothing)\end{array}$ & $\begin{array}{c}\text { Calculated re fraction angle } \\
(\omega)\end{array}$ \\
\hline $0^{\circ}$ & 0 \\
$30^{\circ}$ & $22^{\circ} 02^{\prime}$ \\
$60^{\circ}$ & $40^{\circ} 30^{\prime}$ \\
$75^{\circ}$ & $46^{\circ} 25^{\prime}$ \\
$80^{\circ}$ & $47^{\circ} 37^{\prime}$ \\
$85^{\circ}$ & $48^{\circ} 20^{\prime}$ \\
$90^{\circ}$ & $48^{\circ} 35^{\prime}$ \\
\hline
\end{tabular}

\section{RESULTS}

The total number of lamps and lighting characteristics used by the three selected boats are tabulated in Table 2. Boat A was equipped with 34 lamps; total power generated was 16,700 watts, Boat B with 31 lamps; 15,300 watts and Boat $\mathrm{C}$ with 26 lamps; 12,800 watts. Boat A has the highest power light source among the experimental boats. The average heights of light source for Boat A, Boat B and Boat C were measured to be $3.05,2.80$ and $2.65 \mathrm{~m}$, respectively.

Application of the estimated underwater light distribution for the three selected boats based on the theoretical modeling formula were calculated and shown in Table 3 for Boat A, Table 4 for Boat 
Tabel 2. Lighting characteristics of boats A, B and C

\begin{tabular}{|c|c|c|c|c|c|c|}
\hline Bonts & Types of lamps & $\begin{array}{l}\text { Number } \\
\text { of lamps }\end{array}$ & $\begin{array}{c}\text { Total } \\
\text { wattage }\end{array}$ & $\begin{array}{c}\text { Lighting } \\
\text { efficiency } \\
\text { (lumen/watts) }\end{array}$ & $\begin{array}{l}\text { Total } \\
\text { lumen }\end{array}$ & $\begin{array}{l}\text { Total * } \\
\text { candela }\end{array}$ \\
\hline \multirow{4}{*}{ Bout A } & $\begin{array}{c}\text { Incandescent } \\
(500 \mathrm{w})\end{array}$ & 25 & 12.500 & 19.2 & 240,000 & 19,099 \\
\hline & HP mercury (500w) & 6 & 3,000 & 37.5 & 112,500 & 8,953 \\
\hline & HP mercury (400w) & 3 & 1,200 & 37.5 & 45,000 & 3,581 \\
\hline & Total & 34 & 16,700 & 23.8 & 397,500 & 31.632 \\
\hline \multirow{4}{*}{ Bo at B } & $\begin{array}{c}\text { Incandescent } \\
(500 \mathrm{w})\end{array}$ & 0 & 0 & 0 & 0 & 0 \\
\hline & HP mercury (500w) & 29 & 14,500 & 37.5 & 543,750 & 42,554 \\
\hline & HP mercury (400w) & 2 & 800 & 46.9 & 37,500 & 2,984 \\
\hline & Total & 31 & 15,300 & 38.0 & 581,250 & 46,254 \\
\hline \multirow{4}{*}{ Bo at C } & $\begin{array}{c}\text { Incandescent } \\
(500 \mathrm{w})\end{array}$ & 20 & 10,000 & 19.2 & 192,000 & 15,279 \\
\hline & HP mercury (500w) & 4 & 2,000 & 37.5 & 75.000 & 5.968 \\
\hline & HP mercury (400w) & 2 & 800 & 37.5 & 30,000 & 2,387 \\
\hline & Total & 26 & 12,800 & 23.2 & 297,000 & 23,634 \\
\hline
\end{tabular}

$\mathrm{B}$ and Table 5 for Boat C. Using these values, schematic diagrams of underwater light distribution up to a depth of $40 \mathrm{~m}$ for the boats were drawn and presented in Figures 1, 2 and 3. From the results, it was found that the vertical distance of light transmission in water for the three boats were different. To simplify computation, a $25 \mathrm{~m}$ depth was taken as a reference point and assumed to be the sea bottom (water depth in the fishing ground ranges from 8 $\mathrm{m}$ to $22 \mathrm{~m}$ ). At the depth of $25 \mathrm{~m}$, Boat B was found to have the highest underwater light intensity followed by Boat $\mathrm{A}$ and Boat $\mathrm{C}$ with underwater illuminance values of $4.2,2.9$ and $2.2 \mathrm{lux}$, respectively. It was also observed from the schematic diagrams that the underwater light from the light sources of the three boats managed to reach a depth of more than $40 \mathrm{~m}$ below the sea surface.

\section{DISCUSSION}

This study has presented a simple method for determining the underwater light distribution pattern using a theoretical modeling formula. The results obtained present an approximate value of underwater illuminance due to the influence of factors such as sea condition and fishermen's practice in handling lighting equipment. The main factor that could affect the results is the variation in the actual lumen values of light used under actual field conditions. This is due to the fishermen's practice of occasionally changing the voltage of their electric generators to a higher or lower value than the standard (220-240 volts).

Referring to the illuminance diagrams of Boat A, Boat B and Boat C (Figures 1, 2 and 3), for an isoilluminance of 1 lux as a reference, the depths below the sea surface for Boat A, Boat B and Boat $\mathrm{C}$ are $30.5,32.5$ and $29.0 \mathrm{~m}$ respectively. The result shows that Boat $B$ has the most effective vertical underwater illuminance followed by Boat $A$ and Boat C, even though Boat A possesses the highest power light source among the selected boats (Table 2). It can therefore be concluded that underwater light distribution does not only depend upon the power of the light source (total wattage) but also on the efficiency of the lamp (Table 2). This result differs from the study conducted by Ogura et al. (1985). They concluded from a study on the intensity of light lighted by incandescent lamps for squid lift net fisheries in Thailand that the distance of light in water tends to increase proportionally with the power of light. It was found from this study that even though Boat B had a lower power light source than Boat A, their total lumen is not. This means that the total lumen of light determines underwater illuminance more than the power of light source. On the other hand, Boat B which had a slightly higher power of light than Boat C (2500 watts) produced approximately twice the amount of total lumen. It is thus clear that Boat B was employing the optimum light power compared with the other two boats. This fact is very important to the squid fisheries as fishermen could significantly reduce the cost of the is operations by having an 
Tabel 3. Calculated values of underwater illuminance (lux) of boat A

\begin{tabular}{|c|c|c|c|c|c|c|c|c|c|c|c|c|c|c|c|}
\hline \multirow{2}{*}{ d } & \multicolumn{3}{|c|}{$I A=0^{\circ}$} & \multicolumn{3}{|c|}{$\mid A=15^{\circ}$} & \multicolumn{3}{|c|}{$\mid A=30^{\circ}$} & \multicolumn{3}{|c|}{$\mid A=45^{\circ}$} & \multicolumn{3}{|c|}{$I A=60^{\circ}$} \\
\hline & h & $\mathbf{r}$ & (a) & $h$ & $r$ & (a) & $\mathrm{h}$ & $r$ & (ه & h & $r$ & $t a$ & h & $r$ & Iه \\
\hline 1 & 0 & 1 & 9512.91 & 1.0 & 1.0 & 506.94 & 2.1 & 0.9 & 238.63 & 3.3 & 0.8 & 135.68 & 5.9 & 0.8 & 74.92 \\
\hline 2 & 0 & 2 & 3483.53 & 1.2 & 2.0 & 353.87 & 2.5 & 1.9 & 173.45 & 3.9 & 1.7 & 102.02 & 6.6 & 1.5 & 58.16 \\
\hline 3 & 0 & 3 & 1748.51 & 1.4 & 2.9 & 254.29 & 2.9 & 2.8 & 129.04 & 4.4 & 2.5 & 78.03 & 7.2 & 2.3 & 45.67 \\
\hline 4 & 0 & 4 & 1007.58 & 1.6 & 3.9 & 186.76 & 3.3 & 3.7 & 97.71 & 4.9 & 3.4 & 60.49 & 7.9 & 3.0 & 36.20 \\
\hline 5 & 0 & 5 & 629.02 & 1.8 & 4.9 & 139.54 & 3.6 & 4.6 & 75.03 & 5.5 & 4.2 & 47.40 & 8.5 & 3.8 & 28.92 \\
\hline 6 & 0 & 6 & 414.10 & 2.0 & 5.9 & 105.72 & 4.0 & 5.6 & 58.27 & 6.0 & 5.1 & 37.48 & 9.2 & 4.6 & 23.25 \\
\hline 7 & 0 & 7 & 283.20 & 2.2 & 6.9 & 81.01 & 4.4 & 6.5 & 45.68 & 6.5 & 5.9 & 29.86 & 9.8 & 5.3 & 18.80 \\
\hline 8 & 0 & 8 & 199.34 & 2.4 & 7.8 & 62.69 & 4.8 & 7.4 & 36.01 & 7.1 & 6.8 & 23.94 & 10.5 & 6.1 & 15.28 \\
\hline 9 & 0 & 9 & 143.53 & 2.6 & 8.8 & 48.91 & 5.1 & 8.3 & 27.71 & 7.6 & 7.6 & 19.30 & 11.1 & 6.8 & 12.47 \\
\hline 10 & 0 & 10 & 105.26 & 2.8 & 9.8 & 38.43 & 5.5 & 9.3 & 22.96 & 8.1 & 8.5 & 15.63 & 11.8 & 7.6 & 10.21 \\
\hline 11 & 0 & 11 & 78.37 & 3.0 & 10.8 & 30.39 & 5.9 & 10.2 & 18.47 & 8.6 & 9.3 & 12.72 & 12.4 & 8.4 & 8.39 \\
\hline 12 & 0 & 12 & 59.09 & 3.2 & 11.8 & 24.17 & 6.3 & 11.1 & 14.92 & 9.2 & 10.2 & 10.39 & 13.1 & 9.1 & 6.92 \\
\hline 13 & 0 & 13 & 45.04 & 3.3 & 12.8 & 19.31 & 6.6 & 12.1 & 12.10 & 9.7 & 11.0 & 8.51 & 13.7 & 9.9 & 5.72 \\
\hline 14 & 0 & 14 & 34.66 & 3.5 & 13.7 & 15.50 & 7.0 & 13.0 & 9.85 & 10.2 & 11.9 & 6.99 & 14.4 & 10.6 & 4.74 \\
\hline 15 & 0 & 15 & 26.89 & 3.7 & 14.7 & 12.50 & 7.4 & 13.9 & 8.04 & 10.8 & 12.7 & 5.76 & 15.0 & 11.4 & 3.94 \\
\hline 16 & 0 & 16 & 21.01 & 3.9 & 15.7 & 10.11 & 7.8 & 14.8 & 6.59 & 11.3 & 13.6 & 4.76 & 15.7 & 12.1 & 3.28 \\
\hline 17 & 0 & 17 & 16.52 & 4.1 & 16.7 & 8.20 & 8.1 & 15.8 & 5.41 & 11.8 & 14.4 & 3.94 & 16.3 & 12.9 & 2.73 \\
\hline 18 & 0 & 18 & 13.07 & 4.3 & 17.7 & 6.68 & 8.5 & 16.7 & 4.45 & 12.4 & 15.2 & 3.27 & 17.0 & 13.7 & 2.28 \\
\hline 19 & 0 & 19 & 10.39 & 4.5 & 18.6 & 5.45 & 8.9 & 17.6 & 3.68 & 12.9 & 16.1 & 2.72 & 17.6 & 14.4 & 1.91 \\
\hline 20 & 0 & 20 & 8.29 & 4.7 & 19.6 & 4.46 & 9.3 & 18.5 & 3.04 & 13.4 & 16.9 & 2.27 & 18.3 & 15.2 & 1.60 \\
\hline 21 & 0 & 21 & 6.67 & 4.9 & 20.6 & 3.66 & 9.6 & 19.5 & 2.52 & 14.0 & 17.8 & 1.89 & 18.9 & 15.9 & 1.34 \\
\hline 22 & 0 & 22 & 5.36 & 5.1 & 21.6 & 3.01 & 10 & 20.4 & 2.09 & 14.5 & 18.6 & 1.58 & 19.6 & 16.7 & 1.13 \\
\hline 23 & 0 & 23 & 4.33 & 5.3 & 22.6 & 2.48 & 10.4 & 21.3 & 1.74 & 15.0 & 19.5 & 1.32 & 20.2 & 17.5 & 0.95 \\
\hline 24 & 0 & 24 & 3.51 & 5.5 & 23.5 & 2.05 & 10.8 & 22.3 & 1.45 & 15.6 & 20.3 & 1.11 & 20.9 & 18.2 & 0.80 \\
\hline 25 & 0 & 25 & 2.86 & 5.7 & 24.5 & 1.70 & 11.1 & 23.2 & 1.21 & 16.1 & 21.2 & 0.93 & 21.5 & 19.0 & 0.67 \\
\hline 26 & 0 & 26 & 2.33 & 5.9 & 25.5 & 1.41 & 11.5 & 24.1 & 1.01 & 16.6 & 22.0 & 0.78 & 22.2 & 19.7 & 0.57 \\
\hline 27 & 0 & 27 & 1.91 & 6.1 & 26.5 & 1.17 & 11.9 & 25.0 & 0.84 & 17.1 & 22.9 & 0.66 & 22.9 & 20.5 & 0.48 \\
\hline 28 & 0 & 28 & 1.56 & 6.3 & 27.5 & 0.97 & 12.2 & 26.0 & 0.71 & 17.7 & 23.7 & 0.55 & 23.5 & 21.3 & 0.41 \\
\hline 29 & 0 & 29 & 1.28 & 6.5 & 28.4 & 0.81 & 12.6 & 26.9 & 0.59 & 18.2 & 24.6 & 0.47 & 24.2 & 22.0 & 0.34 \\
\hline 30 & 0 & 30 & 1.05 & 6.7 & 29.4 & 0.67 & 13.0 & 27.8 & 0.50 & 18.7 & 25.4 & 0.39 & 24.8 & 22.8 & 0.29 \\
\hline 31 & 0 & 31 & 0.87 & 6.9 & 30.4 & 0.56 & 13.4 & 28.7 & 0.42 & 19.3 & 26.3 & 0.33 & 25.5 & 23.5 & 0.25 \\
\hline 32 & 0 & 32 & 0.72 & 7.0 & 31.4 & 0.47 & 13.7 & 29.7 & 0.35 & 19.8 & 27.1 & 0.28 & 26.1 & 24.3 & 0.21 \\
\hline 33 & 0 & 33 & 0.60 & 7.2 & 32.4 & 0.39 & 14.1 & 30.6 & 0.30 & 20.3 & 28.0 & 0.24 & 26.8 & 25.1 & 0.18 \\
\hline 34 & 0 & 34 & 0.49 & 7.4 & 33.4 & 0.28 & 14.5 & 31.5 & 0.25 & 20.9 & 28.8 & 0.20 & 27.4 & 25.8 & 0.15 \\
\hline 35 & 0 & 35 & 0.41 & 7.6 & 34.3 & 0.33 & 14.9 & 32.5 & 0.21 & 21.4 & 29.6 & 0.17 & 28.1 & 26.6 & 0.13 \\
\hline 36 & 0 & 36 & 0.34 & 7.8 & 35.3 & 0.23 & 15.2 & 33.4 & 0.18 & 21.9 & 30.5 & 0.14 & 28.7 & 27.3 & 0.11 \\
\hline 37 & 0 & 37 & 0.28 & 8.0 & 36.3 & 0.20 & 15.6 & 34.3 & 0.15 & 22.5 & 31.3 & 0.12 & 29.4 & 28.1 & 0.09 \\
\hline 38 & 0 & 38 & 0.24 & 8.2 & 37.3 & 0.16 & 16.0 & 35.2 & 0.13 & 23.0 & 32.2 & 0.10 & 30.0 & 28.9 & 0.08 \\
\hline 39 & 0 & 39 & 0.20 & 8.4 & 38.3 & 0.14 & 16.4 & 36.2 & 0.11 & 23.5 & 33.0 & 0.09 & 30.7 & 29.6 & 0.07 \\
\hline 40 & 0 & 40 & 0.17 & 8.6 & 39.2 & 0.12 & 16.7 & 37.1 & 0.09 & 24.1 & 33.9 & 0.08 & 31.3 & 30.4 & 0.06 \\
\hline
\end{tabular}


Tabel 4. Calculated values of underwater illuminance (lux) of boat B

\begin{tabular}{|c|c|c|c|c|c|c|c|c|c|c|c|c|c|c|c|}
\hline \multirow{2}{*}{ d } & \multicolumn{3}{|c|}{$\mid A=0^{\circ}$} & \multicolumn{3}{|c|}{$\mid A=15^{\circ}$} & \multicolumn{3}{|c|}{$\mid A=30^{\circ}$} & \multicolumn{3}{|c|}{$\mid A=45$} & \multicolumn{3}{|c|}{$1 A=60^{\circ}$} \\
\hline & h & $r$ & ID & h & $r$ & 10 & h & $r$ & 10 & h & $\mathbf{r}$ & 100 & h & $r$ & 10 \\
\hline 1 & 0 & 1 & 14889.37 & 1.0 & 1.0 & 860.56 & 2.1 & 0.9 & 406.68 & 3.3 & 0.8 & 231.99 & 5.9 & 0.8 & 129.98 \\
\hline 2 & 0 & 2 & 5389.69 & 1.2 & 2.0 & 591.38 & 2.5 & 1.9 & 291.76 & 3.9 & 1.7 & 172.53 & 6.6 & 1.5 & 100.92 \\
\hline 3 & 0 & 3 & 2683.19 & 1.4 & 2.9 & 419.89 & 2.9 & 2.8 & 214.90 & 4.4 & 2.5 & 130.82 & 7.2 & 2.3 & 79.25 \\
\hline 4 & 0 & 4 & 1536.76 & 1.6 & 3.9 & 305.45 & 3.3 & 3.7 & 161.43 & 4.9 & 3.4 & 100.69 & 7.9 & 3.0 & 62.81 \\
\hline 5 & 0 & 5 & 954.84 & 1.8 & 4.9 & 226.42 & 3.6 & 4.6 & 123.13 & 5.5 & 4.2 & 78.44 & 8.5 & 3.8 & 50.17 \\
\hline 6 & 0 & 6 & 626.22 & 2.0 & 5.9 & 170.39 & 4.0 & 5.6 & 95.08 & 6.0 & 5.1 & 61.70 & 9.2 & 4.6 & 40.34 \\
\hline 7 & 0 & 7 & 426.95 & 2.2 & 6.9 & 129.82 & 4.4 & 6.5 & 74.17 & 6.5 & 5.9 & 48.94 & 9.8 & 5.3 & 32.62 \\
\hline 8 & 0 & 8 & 299.76 & 2.4 & 7.8 & 99.95 & 4.8 & 7.4 & 58.35 & 7.1 & 6.8 & 39.08 & 10.5 & 6.1 & 26.50 \\
\hline 9 & 0 & 9 & 215.37 & 2.6 & 8.8 & 77.64 & 5.1 & 8.3 & 46.23 & 7.6 & 7.6 & 31.40 & 11.1 & 6.8 & 21.63 \\
\hline 10 & 0 & 10 & 157.64 & 2.8 & 9.8 & 60.77 & 5.5 & 9.3 & 36.86 & 8.1 & 8.5 & 25.35 & 11.8 & 7.6 & 17.72 \\
\hline 11 & 0 & 11 & 117.18 & 3.0 & 10.8 & 47.88 & 5.9 & 10.2 & 39.55 & 8.6 & 9.3 & 20.58 & 12.4 & 8.4 & 14.56 \\
\hline 12 & 0 & 12 & 88.23 & 3.2 & 11.8 & 37.95 & 6.3 & 11.1 & 23.81 & 9.2 & 10.2 & 16.75 & 13.1 & 9.1 & 12.01 \\
\hline 13 & 0 & 13 & 67.17 & 3.3 & 12.8 & 30.24 & 6.6 & 12.1 & 19.25 & 9.7 & 11.0 & 13.69 & 13.7 & 9.9 & 9.93 \\
\hline 14 & 0 & 14 & 51.63 & 3.5 & 13.7 & 24.21 & 7.0 & 13.0 & 15.63 & 10.2 & 11.9 & 11.23 & .14 .4 & 10.6 & 8.23 \\
\hline 15 & 0 & 15 & 40.01 & 3.7 & 14.7 & 19.47 & 7.4 & 13.9 & 12.74 & 10.8 & 12.7 & 9.23 & 15.0 & 11.4 & 6.83 \\
\hline 16 & 0 & 16 & 31.24 & 3.9 & 15.7 & 15.71 & 7.8 & 14.8 & 10.41 & 11.3 & 13.6 & 7.61 & 15.7 & 12.1 & 5.69 \\
\hline 17 & 0 & 17 & 24.55 & 4.1 & 16.7 & 12.72 & 8.1 & 15.8 & 8.53 & 11.8 & 14.4 & 6.29 & 16.3 & 12.9 & 4.74 \\
\hline 18 & 0 & 18 & 19.40 & 4.3 & 17.7 & 10.34 & 8.5 & 16.7 & 7.01 & 12.4 & 15.2 & 5.21 & 17.0 & 13.7 & 3.96 \\
\hline 19 & 0 & 19 & 15.41 & 4.5 & 18.6 & 8.43 & 8.9 & 17.6 & 5.78 & 12.9 & 16.1 & 4.33 & 17.6 & 14.4 & 3.31 \\
\hline 20 & 0 & 20 & 12.30 & 4.7 & 19.6 & 6.89 & 9.3 & 18.5 & 4.77 & 13.4 & 16.9 & 3.60 & 18.3 & 15.2 & 2.78 \\
\hline 21 & 0 & 21 & 9.86 & 4.9 & 20.6 & 5.64 & 9.6 & 19.5 & 3.94 & 14.0 & 17.8 & 3.00 & 18.9 & 15.9 & 2.33 \\
\hline 22 & 0 & 22 & 7.94 & 5.1 & 21.6 & 4.63 & 10 & 20.4 & 3.27 & 14.5 & 18.6 & 2.50 & 19.6 & 16.7 & 1.96 \\
\hline 23 & 0 & 23 & 6.41 & 5.3 & 226 & 3.81 & 10.4 & 21.3 & 2.71 & 15.0 & 19.5 & 2.09 & 20.2 & 17.5 & 1.65 \\
\hline 24 & 0 & 24 & 5.20 & 5.5 & 23.5 & 3.14 & 10.8 & 22.3 & 2.26 & 15.6 & 20.3 & 1.75 & 20.9 & 18.2 & 1.39 \\
\hline 25 & 0 & 25 & 4.22 & 5.7 & 24.5 & 2.60 & 11.1 & 23.2 & 1.88 & 16.1 & 21.2 & 1.46 & 21.5 & 19.0 & 1.17 \\
\hline 26 & 0 & 26 & 3.44 & 5.9 & 25.5 & 2.15 & 11.5 & 24.1 & 1.57 & 16.6 & 22.0 & 1.23 & 22.2 & 19.7 & 0.99 \\
\hline 27 & 0 & 27 & 2.81 & 6.1 & 26.5 & 1.78 & 11.9 & 25.0 & 1.31 & 17.1 & 22.9 & 1.03 & 22.9 & 20.5 & 0.83 \\
\hline 28 & 0 & 28 & 230 & 6.3 & 27.5 & 1.48 & 12.2 & 26.0 & 1.10 & 17.7 & 23.7 & 0.87 & 23.5 & 21.3 & 0.71 \\
\hline 29 & 0 & 29 & 1.89 & 6.5 & 28.4 & 1.23 & 12.6 & 26.9 & 0.92 & 18.2 & 24.6 & 0.73 & 24.2 & 22.0 & 0.60 \\
\hline 30 & 0 & 30 & 1.56 & 6.7 & 29.4 & 1.03 & 13.0 & 27.8 & 0.77 & 18.7 & 25.4 & 0.62 & 24.8 & 22.8 & 0.51 \\
\hline 31 & 0 & 31 & 1.28 & 6.9 & 30.4 & 0.86 & 13.4 & 28.7 & 0.65 & 19.3 & 26.3 & 0.52 & 25.5 & 23.5 & 0.43 \\
\hline 32 & 0 & 32 & 1.06 & 7.0 & 31.4 & 0.71 & 13.7 & 29.7 & 0.54 & 19.8 & 27.1 & 0.44 & 26.1 & 24.3 & 0.36 \\
\hline 33 & 0 & 33 & 0.88 & 7.2 & 32.4 & 0.60 & 14.1 & 30.6 & 0.46 & 20.3 & 28.0 & 0.37 & 26.8 & 25.1 & 0.31 \\
\hline 34 & 0 & 34 & 073 & 7.4 & 33.4 & 0.50 & 14.5 & 31.5 & 0.38 & 20.9 & 28.8 & 0.31 & 27.4 & 25.8 & 0.26 \\
\hline 35 & 0 & 35 & 0.60 & 7.6 & 34.3 & 0.42 & 14.9 & 32.5 & 0.32 & 21.4 & 29.6 & 0.27 & 28.1 & 26.6 & 0.22 \\
\hline 36 & 0 & 36 & 0.50 & 7.8 & 35.3 & 0.35 & 15.2 & 33.4 & 0.27 & 21.9 & 30.5 & 0.22 & 28.7 & 27.3 & 0.19 \\
\hline 37 & 0 & 37 & 0.42 & 8.0 & 36.3 & 0.30 & 15.6 & 34.3 & 0.23 & 22.5 & 31.3 & 0.19 & 29.4 & 28.1 & 0.16 \\
\hline 38 & 0 & 38 & 0.35 & 8.2 & 37.3 & 0.25 & 16.0 & 35.2 & 0.19 & 23.0 & 32.2 & 0.16 & 30.0 & 28.9 & 0.14 \\
\hline 39 & 0 & 39 & 0.29 & 8.4 & 38.3 & 0.21 & 16.4 & 36.2 & 0.16 & 23.5 & 33.0 & 0.14 & 30.7 & 29.6 & 0.12 \\
\hline 40 & 0 & 40 & 0.24 & 8.6 & 39.2 & 0.18 & 16.7 & 37.1 & 0.14 & 24.1 & 33.9 & 0.12 & 31.3 & 30.4 & 0.10 \\
\hline
\end{tabular}

Key:

$\mathrm{IA}=$ Incident Angle $\left({ }^{\circ}\right.$ )

$\mathrm{d}=$ Distance of destination of light in water $(\mathrm{m})$

$\mathrm{h}=$ Horizontal distance $(\mathrm{m})$

$r \quad=$ Vertical distance $(m)$

$|\omega|=$ Illuminance (lux) 
Tabel 5. Calculated values of underwater illuminance (lux) of boat C

\begin{tabular}{|c|c|c|c|c|c|c|c|c|c|c|c|c|c|c|c|}
\hline \multirow{2}{*}{$d$} & \multicolumn{3}{|c|}{$I A=0^{\circ}$} & \multicolumn{3}{|c|}{$I A=15^{\circ}$} & \multicolumn{3}{|c|}{$I A=30^{\circ}$} & \multicolumn{3}{|c|}{$I A=45^{\circ}$} & \multicolumn{3}{|c|}{$I A=60^{\circ}$} \\
\hline & h & $r$ & 10 & $\mathrm{~h}$ & $r$ & la & h & $r$ & I & $h$ & $r$ & 10 & h & $r$ & ta \\
\hline 1 & 0 & 1 & 7943.35 & 1.0 & 1.0 & 483.64 & 2.1 & 0.9 & 229.16 & 3.3 & 0.8 & 131.03 & 5.9 & 0.8 & 73.61 \\
\hline 2 & 0 & 2 & 2853.37 & 1.2 & 2.0 & 328.89 & 2.5 & 1.9 & 162.98 & 3.9 & 1.7 & 96.72 & 6.6 & 1.5 & 56.82 \\
\hline 3 & 0 & 3 & 1412.93 & 1.4 & 2.9 & 231.67 & 2.9 & 2.8 & 119.25 & 4.4 & 2.5 & 72.91 & 7.2 & 2.3 & 44.41 \\
\hline 4 & 0 & 4 & 806.04 & 1.6 & 3.9 & 167.43 & 3.3 & 3.7 & 89.11 & 4.9 & 3.4 & 55.86 & 7.9 & 3.0 & 35.06 \\
\hline 5 & 0 & 5 & 499.30 & 1.8 & 4.9 & 123.50 & 3.6 & 4.6 & 67.67 & 5.5 & 4.2 & 43.35 & 8.5 & 3.8 & 27.91 \\
\hline 6 & 0 & 6 & 326.68 & 2.0 & 5.9 & 92.54 & 4.0 & 5.6 & 52.07 & 6.0 & 5.1 & 33.99 & 9.2 & 4.6 & 22.37 \\
\hline 7 & 0 & 7 & 222.29 & 2.2 & 6.9 & 70.24 & 4.4 & 6.5 & 40.49 & 6.5 & 5.9 & 26.88 & 9.8 & 5.3 & 18.04 \\
\hline 8 & 0 & 8 & 155.82 & 2.4 & 7.8 & 53.91 & 4.8 & 7.4 & 31.76 & 7.1 & 6.8 & 21.41 & 10.5 & 6.1 & 14.63 \\
\hline 9 & 0 & 9 & 111.80 & 2.6 & 8.8 & 41.75 & 5.1 & 8.3 & 25.10 & 7.6 & 7.6 & 17.16 & 11.1 & 6.8 & 11.91 \\
\hline 10 & 0 & 10 & 81.74 & 2.8 & 9.8 & 32.57 & 5.5 & 9.3 & 19.97 & 8.1 & 8.5 & 13.83 & 11.8 & 7.6 & 9.74 \\
\hline 11 & 0 & 11 & 60.70 & 3.0 & 10.8 & 25.63 & 5.9 & 10.2 & 15.98 & 8.6 & 9.3 & 11.20 & 12.4 & 8.4 & 7.99 \\
\hline 12 & 0 & 12 & 45.66 & 3.2 & 11.8 & 20.27 & 6.3 & 11.1 & 12.84 & 9.2 & 10.2 & 9.10 & 13.1 & 9.1 & 6.58 \\
\hline 13 & 0 & 13 & 34.74 & 3.3 & 12.8 & 16.12 & 6.6 & 12.1 & 10.37 & 9.7 & 11.0 & 7.43 & 13.7 & 9.9 & 5.43 \\
\hline 14 & 0 & 14 & 26.68 & 3.5 & 13.7 & 12.88 & 7.0 & 13.0 & 8.41 & 10.2 & 11.9 & 6.08 & 14.4 & 10.6 & 4.49 \\
\hline 15 & 0 & 15 & 20.67 & 3.7 & 14.7 & 10.34 & 7.4 & 13.9 & 6.84 & 10.8 & 12.7 & 5.00 & 15.0 & 11.4 & 3.73 \\
\hline 16 & 0 & 16 & 16.12 & 3.9 & 15.7 & 8.33 & 7.8 & 14.8 & 5.58 & 11.3 & 13.6 & 4.11 & 15.7 & 12.1 & 3.10 \\
\hline 17 & 0 & 17 & 12.66 & 4.1 & 16.7 & 6.74 & 8.1 & 15.8 & 4.57 & 11.8 & 14.4 & 3.40 & 16.3 & 12.9 & 2.58 \\
\hline 18 & 0 & 18 & 10.00 & 4.3 & 17.7 & 5.47 & 8.5 & 16.7 & 3.75 & 124 & 15.2 & 2.81 & 17.0 & 13.7 & 2.15 \\
\hline 19 & 0 & 19 & 7.95 & 4.5 & 18.6 & 4.45 & 8.9 & 17.6 & 3.09 & 12.9 & 16.1 & 2.33 & 17.6 & 14.4 & 1.80 \\
\hline 20 & 0 & 20 & 6.34 & 4.7 & 19.6 & 3.64 & 9.3 & 18.5 & 2.55 & 13.4 & 16.9 & 1.94 & 18.3 & 15.2 & 1.51 \\
\hline 21 & 0 & 21 & 5.08 & 4.9 & 20.6 & 2.98 & 9.6 & 19.5 & 2.10 & 14.0 & 17.8 & 1.61 & 18.9 & 15.9 & 1.26 \\
\hline 22 & 0 & 22 & 4.09 & 5.1 & 21.6 & 2.44 & 10 & 20.4 & 1.74 & 14.5 & 18.6 & 1.34 & 19.6 & 16.7 & 1.06 \\
\hline 23 & 0 & 23 & 3.30 & 5.3 & 22.6 & 2.01 & 10.4 & 21.3 & 1.44 & 15.0 & 19.5 & 1.12 & 20.2 & 17.5 & 0.89 \\
\hline 24 & 0 & 24 & 2.67 & 5.5 & 23.5 & 1.65 & 10.8 & 22.3 & 1.20 & 15.6 & 20.3 & 0.94 & 20.9 & 18.2 & 0.75 \\
\hline 25 & 0 & 25 & 2.17 & 5.7 & 24.5 & 1.37 & 11.1 & 23.2 & 1.00 & 16.1 & 21.2 & 0.78 & 21.5 & 19.0 & 0.63 \\
\hline 26 & 0 & 26 & 1.77 & $5: 9$ & 25.5 & 1.13 & 11.5 & 24.1 & 0.83 & 16.6 & 22.0 & 0.66 & 22.2 & 19.7 & 0.53 \\
\hline 27 & 0 & 27 & 1.45 & 6.1 & 26.5 & 0.94 & 11.9 & 250 & 0.70 & 17.1 & 22.9 & 0.55 & 22.9 & 20.5 & 0.45 \\
\hline 28 & 0 & 28 & 1.19 & 6.3 & 27.5 & 0.78 & 12.2 & 26.0 & 0.58 & 17.7 & 23.7 & 0.46 & 23.5 & 21.3 & 0.38 \\
\hline 29 & 0 & 29 & 0.97 & 6.5 & 28.4 & 0.65 & 12.6 & 26.9 & 0.49 & 18.2 & 24.6 & 0.39 & 24.2 & 22.0 & 0.32 \\
\hline 30 & 0 & 30 & 0.80 & 6.7 & 29.4 & 0.54 & 13.0 & 27.8 & 0.41 & 18.7 & 25.4 & 0.33 & 24.8 & 22.8 & 0.27 \\
\hline 31 & 0 & 31 & 0.66 & 6.9 & 30.4 & 0.45 & 13.4 & 28.7 & 0.34 & 19.3 & 26.3 & 0.28 & 25.5 & 23.5 & 0.23 \\
\hline 32 & 0 & 32 & 0.55 & 7.0 & 31.4 & 0.37 & 13.7 & 29.7 & 0.29 & 19.8 & 27.1 & 0.23 & 26.1 & 24.3 & 0.20 \\
\hline 33 & 0 & 33 & 0.45 & 7.2 & 32.4 & 0.31 & 14.1 & 30.6 & 0.24 & 20.3 & 28.0 & 0.20 & 26.8 & 25.1 & 0.17 \\
\hline 34 & 0 & 34 & 0.37 & 7.4 & 33.4 & 0.26 & 14.5 & 31.5 & 0.20 & 20.9 & 28.8 & 0.17 & 27.4 & 25.8 & 0.14 \\
\hline 35 & 0 & 35 & 0.31 & 7.6 & 34.3 & 0.22 & 14.9 & 32.5 & 0.17 & 21.4 & 29.6 & 0.14 & 28.1 & 26.6 & 0.12 \\
\hline 36 & 0 & 36 & 0.26 & 7.8 & 35.3 & 0.18 & 15.2 & 33.4 & 0.14 & 21.9 & 30.5 & 0.12 & 28.7 & 27.3 & 0.10 \\
\hline 37 & 0 & 37 & 0.22 & 8.0 & 36.3 & 0.15 & 15.6 & 34.3 & 0.12 & 22.5 & 31.3 & 0.10 & 29.4 & 28.1 & 0.09 \\
\hline 38 & 0 & 38 & 0.18 & 8.2 & 37.3 & 0.13 & 16.0 & 35.2 & 0.10 & 23.0 & 32.2 & 0.09 & 30.0 & 28.9 & 0.07 \\
\hline 39 & 0 & 39 & 0.15 & 8.4 & 38.3 & 0.11 & 16.4 & 36.2 & 0.09 & 23.5 & 33.0 & 0.07 & 30.7 & 29.6 & 0.06 \\
\hline 40 & 0 & 40 & 0.13 & 8.6 & 39.2 & 0.09 & 16.7 & 37.1 & 0.07 & 24.1 & 33.9 & 0.06 & 31.3 & 30.4 & 0.05 \\
\hline
\end{tabular}

\footnotetext{
Key:

$\mathrm{A}=$ Incident Angle $\left(^{\circ}\right.$ )

$d=$ Distance of destination of light in water $(\mathrm{m})$

$h=$ Horizontal distance $(m)$

$r=$ Vertical distance $(m)$

(D) $=$ Illuminance (lux)
} 
IFR Journal Vol. IV No.1, 1998

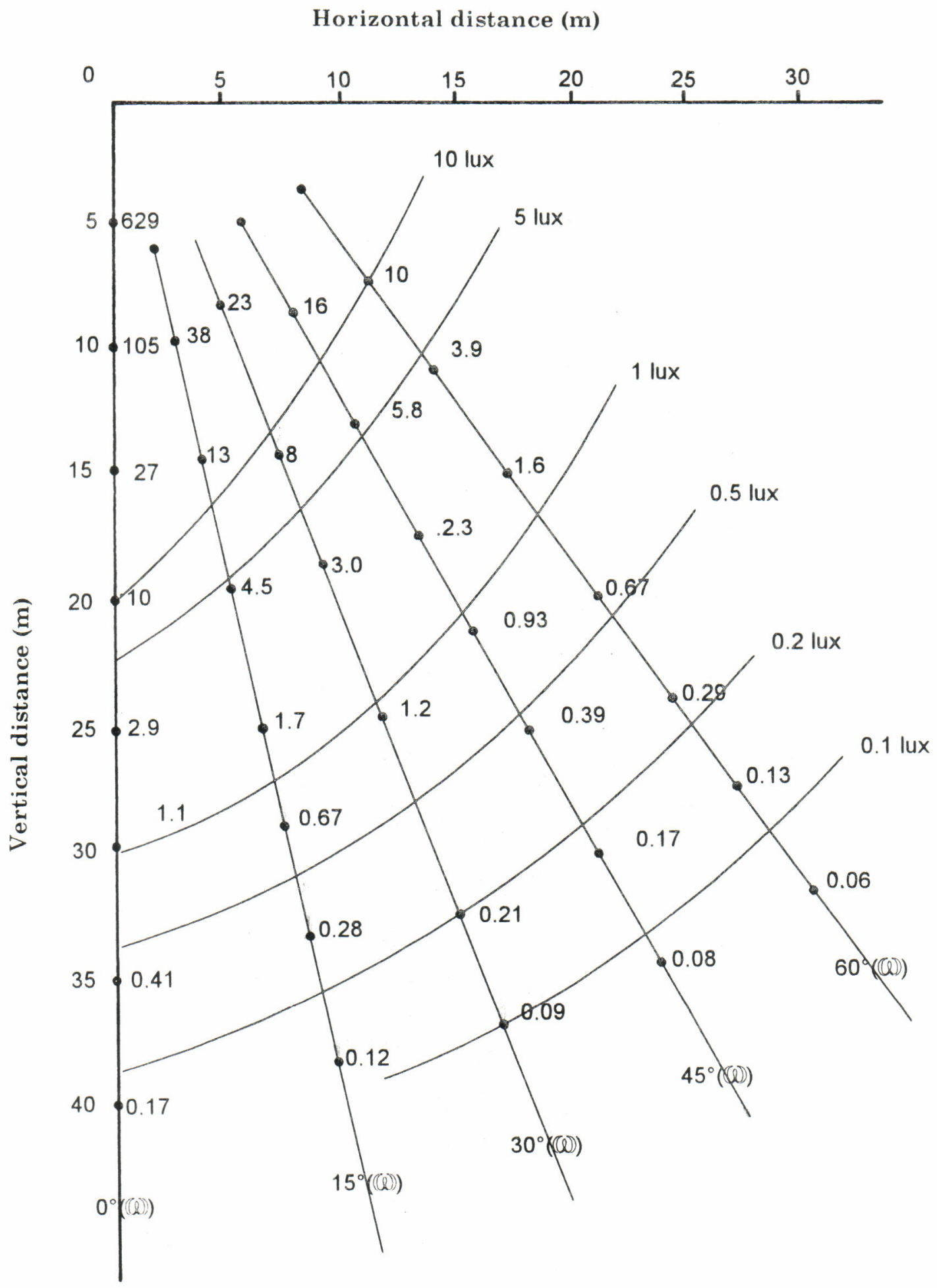

Figure 1. Schematic diagram of distribution pattern of underwater illuminance employed by boat A. 
S. Ibrahim, A.M. Ambak, K. Samo, M.Z. Zakaria, Z.A. Shahardin and S. Hajisamae

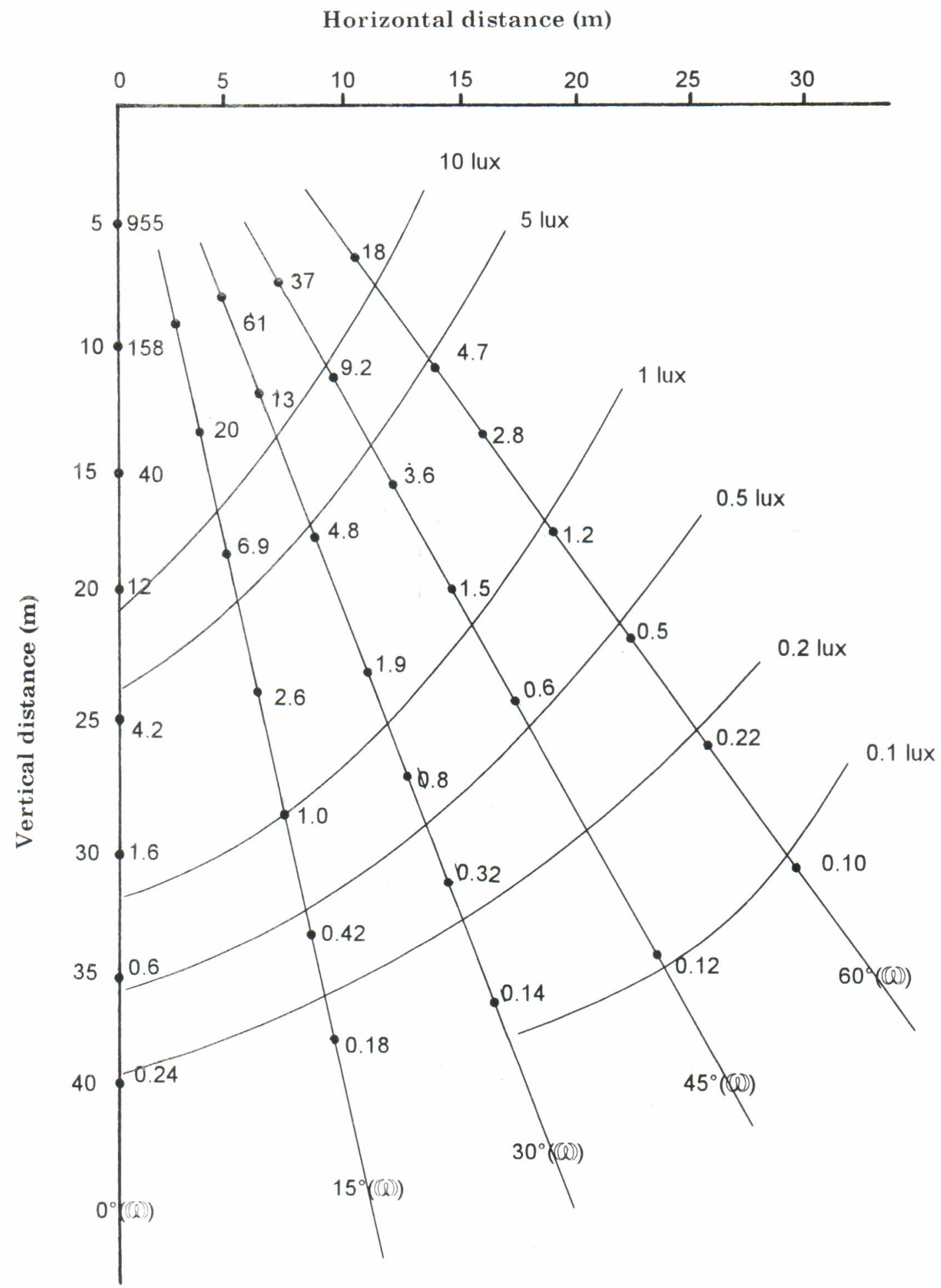

Figure 2. Schematic diagram of distribution pattern of underwater illuminance employed by boat B. 


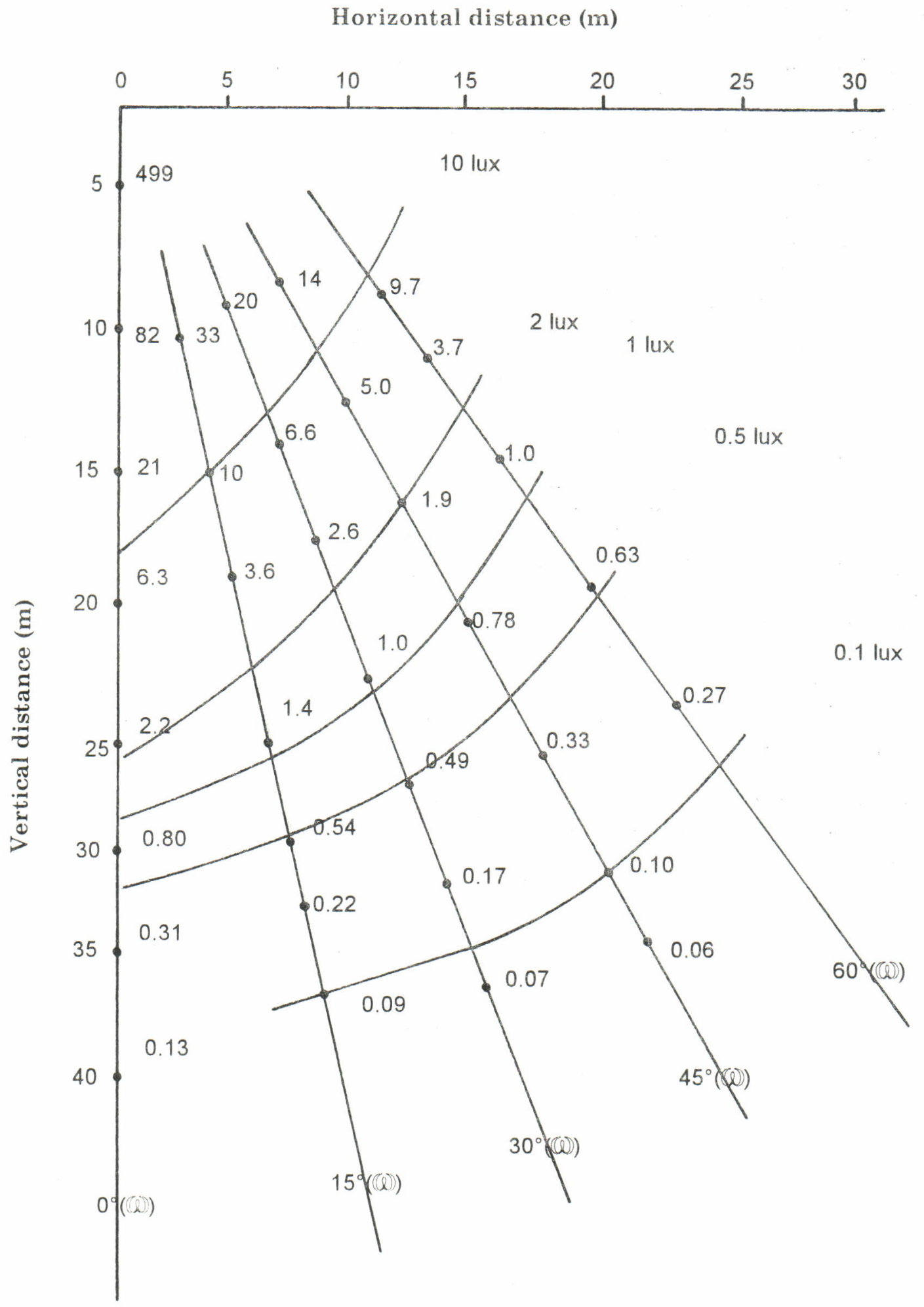

Figure 3. Schematic diagram of distribution pattern of underwater illuminance employed by boat C. 
appropriate lighting system. It would also enable squid fishermen to have selective fishing based on the preferences of the squid species for underwater light illuminance.

This study also reveals that the squid netting boats in Terengganu waters have been using excess power in their attracting light system. Hajisamae (1996) noted that the minimum preference level of underwater light illuminance for Sepioteuthis lessoniana and Loligo chinensis is only 1.5 lux. However, it was found from this study that at a depth of $25 \mathrm{~m}$, Boat $\mathrm{C}$ recorded an underwater light intensity of 2.2 lux. This shows that, even though Boat $\mathrm{C}$ employed the smallest power of lighting system, it was still excessively powered and illuminated beyond the $25 \mathrm{~m}$ depth. From the results of this study, it is recommended that high pressure mercury lamps be used for the squid net fishery as they provide larger lighting efficiencies, longer life span and lower energy consumption as compared to the incandescent lamps. This is supported by the fact that there is no significant difference in daily CPUE (Catch Per Unit Effort) between squid fishing boats using high pressure mercury lamps and those using incandescent lamps as the main light attracting system (Hajisamae, 1996).

\section{ACKNOWLEDGMENTS}

The authors wish to thank Universiti Putra Malaysia for the financial support and the squid fishermen in the study area for providing relevant information.

\section{REFERENCES}

Ashirin, Z.S. and Ibrahim, A.R. 1992. Preliminary Squid Survey in Coastal Waters off Kuala Terengganu, Malaysia. Asian Fish. Sci. 5(1992): $261 \cdot 264$.

Hamid, A.S. 1990. Comparative Study On Catching Squid With Light Attraction. Master's thesis, Tokyo University of Fisheries, Tokyo, Japan.

Hayase, S. Miyata, C. Yamazaki, T. Narintharanggura, S., Supongpun, S. and Saikliane, P. 1983. Preliminary Study on the Estimating Effective Light Intensity for Purse Seine Fisheries in Thailand. SEAFDEC Current Tech. Pap Ser. No. 8.

Kawamura, G., Moninja, D.R. and Mangunsukarto, K. 1983. Fishing Gears and Methods in Java, Madura and Bali Islands, Indonesia. Rep. Kagoshima Univ. Sci. Sur. p. 9-40.

Nomura, M. 1985. Fishing Techniques (3). Japan International Cooperation Agency (JICA), Tokyo. Japan. p 90-96

Ogura, M., Arimoto, T. and Hayase, S. 1985. Intensity of Underwater Light for Squid Lift-Net Fisheries in Thailand. MP/No.14, SEAFDEC. Bangkok, Thailand.

Sakri, I., Ambak, M.A. and Hajisamae, S. 1995. Fishing Methods for Cephalopods in Malaysia. INFOFISH Int. 1(95):57-60.

Hajisamae, S. 1996. Effect of Light on Squid Behavior and its Application for Squid Net Fishing in Malaysia. Master Thesis, Universiti Putra Malaysia. Serdang, Selangor, Malaysia. 117 pp. 